\title{
Efeito do uso de álcool em pessoas vivendo com HIV/aids: revisão integrativa
}

\author{
Vanessa da Frota Santos ${ }^{1}$, \\ Gilmara Holanda da Cunha², \\ Marli Teresinha Gimeniz Galvão3, \\ Ivana Cristina Vieira de Lima4, \\ Elucir Gir ${ }^{5}$ \\ Ana Karoline Bastos Costa ${ }^{6}$
}

\section{RESUMO}

Estudo objetivou analisar as produções científicas sobre o efeito do uso de álcool em pessoas com HIV/aids. Tratase de uma revisão integrativa realizada nas bases de dados LILACS, SciELO, MEDLINE, CINAHL, SCOPUS, COCHRANE, Web of Science em outubro de 2017. Utilizaram-se os descritores Síndrome da Imunodeficiência Adquirida, HIV e Álcool, em português, inglês e espanhol, com o operador booleano AND. Foram encontrados 2.355 artigos e selecionados 46 . Os dados obtidos foram organizados em quatro categorias temáticas: Efeitos do álcool na adesão à terapia antirretroviral; Efeitos neurológicos e metabólicos do álcool; Aumento do risco de transmissão do HIV e Aumento da progressão da doença. Conclui-se que a comorbidade HIV/aids e o consumo de álcool têm efeito sinérgico na vida de pessoas com HIV, causando maiores danos à saúde, com destaque para as alterações neurológicas e metabólicas, baixa adesão ao tratamento, aumento da transmissão do vírus e progressão da doença.

Descritores: HIV; Etanol; Síndrome de Imunodeficiência Adquirida.

\footnotetext{
${ }^{1}$ Enfermeira, Mestre em Enfermagem. Discente do Programa de Pós-Graduação em Enfermagem, nível Doutorado, da Universidade Federal do Ceará. Professora da Faculdade Ateneu. Fortaleza, CE, Brasil. E-mail: doutorandavanessasantos@outlook.com.

2 Enfermeira, Doutora em Enfermagem. Professora Adjunta da Universidade Federal do Ceará. Fortaleza, CE, Brasil. E-mail:

gilmaraholandaufc@yahoo.com.br.

${ }^{3}$ Enfermeira, Doutora em Doenças Tropicais. Professora Associada da Universidade Federal do Ceará. Fortaleza, CE, Brasil. E-mail: marligalvao@gmail.com.

${ }^{4}$ Enfermeira, Doutora em Enfermagem. Professora Substituta da Universidade Estadual do Ceará. Fortaleza, CE, Brasil. E-mail: doutorandaivana@gmail.com.

${ }^{5}$ Enfermeira, Doutora em Enfermagem. Professora Titular da Escola de Enfermagem de Ribeirão Preto da Universidade de São Paulo. Ribeirão Preto, SP, Brasil. E-mail: egir@eerp.usp.br.

${ }^{6}$ Acadêmica do curso de Graduação em Enfermagem da Universidade Federal do Ceará. Fortaleza, CE, Brasil. E-mail: anakaroline14@hotmail.com.
}

Artigo recebido: 02/05/2017.

Artigo aprovado: 03/04/2018.

Artigo publicado: 31/12/2018.

\section{Como citar esse artigo:}

Santos VF, Cunha GH, Galvão MTG, Lima ICV, Gir E, Costa AKB. Efeito do uso de álcool em pessoas vivendo com HIV/aids: revisão integrativa. Rev. Eletr. Enf. [Internet]. 2018 [acesso em: ];20:v20a42. Disponível em: https://doi.org/10.5216/ree.v20.46712. 


\section{INTRODUÇÃO}

Apesar dos avanços para redução da propagação do Vírus da Imunodeficiência Adquirida (HIV), o número de pessoas acometidas continua em ascensão em todo o mundo ${ }^{(1)}$. Associado a isso, observa-se o uso abusivo de álcool nessa população, um sério problema de saúde, com repercussões sociais, econômicas e psicológicas ${ }^{(2)}$.

Com a utilização da Terapia Antirretroviral (TARV), houve redução significativa na morbidade e mortalidade associadas ao HIV, tornando-se uma doença de característica crônica. Esse fato acarretou o aumento da perspectiva de vida e a suscetibilidade à aquisição de hábitos de risco, como o consumo de álcool, que tende a ser mais prevalente em Pessoas Vivendo com HIV/aids (PVHA), quando comparado à população em geral(1-2), estimando-se que cerca de $44,0 \%$ desses indivíduos tenham problemas com uso do álcool e outras drogas ${ }^{(3)}$.

O consumo de álcool influencia negativamente a condição de saúde das $\mathrm{PVHA}^{(4)}$ e está associado a relações sexuais sem preservativo $^{(1)}$, aumentando a probabilidade de transmissão do HIV e de outras Infecções Sexualmente Transmissíveis (IST) ${ }^{(2)}$. Além disso, pode comprometer a qualidade de vida e a adesão ao tratamento, com aceleração da progressão da doença ${ }^{(3)}$.

Diante do exposto, os profissionais de saúde, em especial o enfermeiro, necessitam conhecer os efeitos do uso do álcool em PVHA, com vistas a prevenir ou minorar os danos associados a essa substância, a partir do estímulo à aquisição de hábitos de vida saudáveis e ao autocuidado.

Com o intuito de contribuir para a assistência em saúde às PVHA, propôs-se este estudo que tem por objetivo analisar as produções científicas acerca do efeito do uso de álcool em pessoas com HIV/aids.

\section{MÉTODO}

Trata-se de uma revisão integrativa de literatura desenvolvida nas seguintes etapas: Elaboração da pergunta norteadora, Busca na literatura, Coleta de dados, Análise crítica dos resultados, Discussão dos resultados e Apresentação da revisão integrativa(5).

Buscou-se responder à seguinte questão norteadora, formulada com base na estratégia PICO(6): "Qual o efeito do uso de álcool em pessoas com HIV/aids?". Os critérios de inclusão consistiram em artigos completos, disponíveis eletronicamente, nos idiomas português, inglês e espanhol, sem recorte temporal. Foram excluídas as publicações repetidas, as quais foram agrupadas na base de dados que continha mais artigos, aquelas que não responderam à questão de pesquisa e os estudos com animais.

A busca eletrônica foi realizada por dois revisores de forma simultânea no mês de outubro de 2017, em cinco bases de dados: Cumulative Index to Nursing and Allied Health Literature (CINAHL), Literatura LatinoAmericana e do Caribe em Ciências da Saúde (LILACS), Scientific Eletronic Library Online (SciELO), Web of Science e SCOPUS; um portal Medical Literature Analysis and Retrieval System Online (MEDLINE/Pubmed) e uma biblioteca COCHRANE. Utilizaram-se os descritores "Síndrome da Imunodeficiência Adquirida", "HIV" e "Álcool", disponíveis no Medical Subject Headings (MeSH) da National Library of Medicine e Descritores em Ciências da Saúde (DeCS), nos idiomas português, inglês e espanhol, com uso do operador booleano AND.

A partir dos cruzamentos realizados foram encontrados 2.355 artigos, sendo selecionados 46 . Foram excluídas 24 publicações duplicadas e 2.285 artigos que abordavam a relação entre o uso do álcool e outras drogas 
em pessoas não infectadas e comportamentos de risco para aquisição do HIV. A Figura 1 mostra a identificação, seleção e inclusão das produções científicas.

Figura 1: Fluxograma de identificação, seleção e inclusão dos estudos.

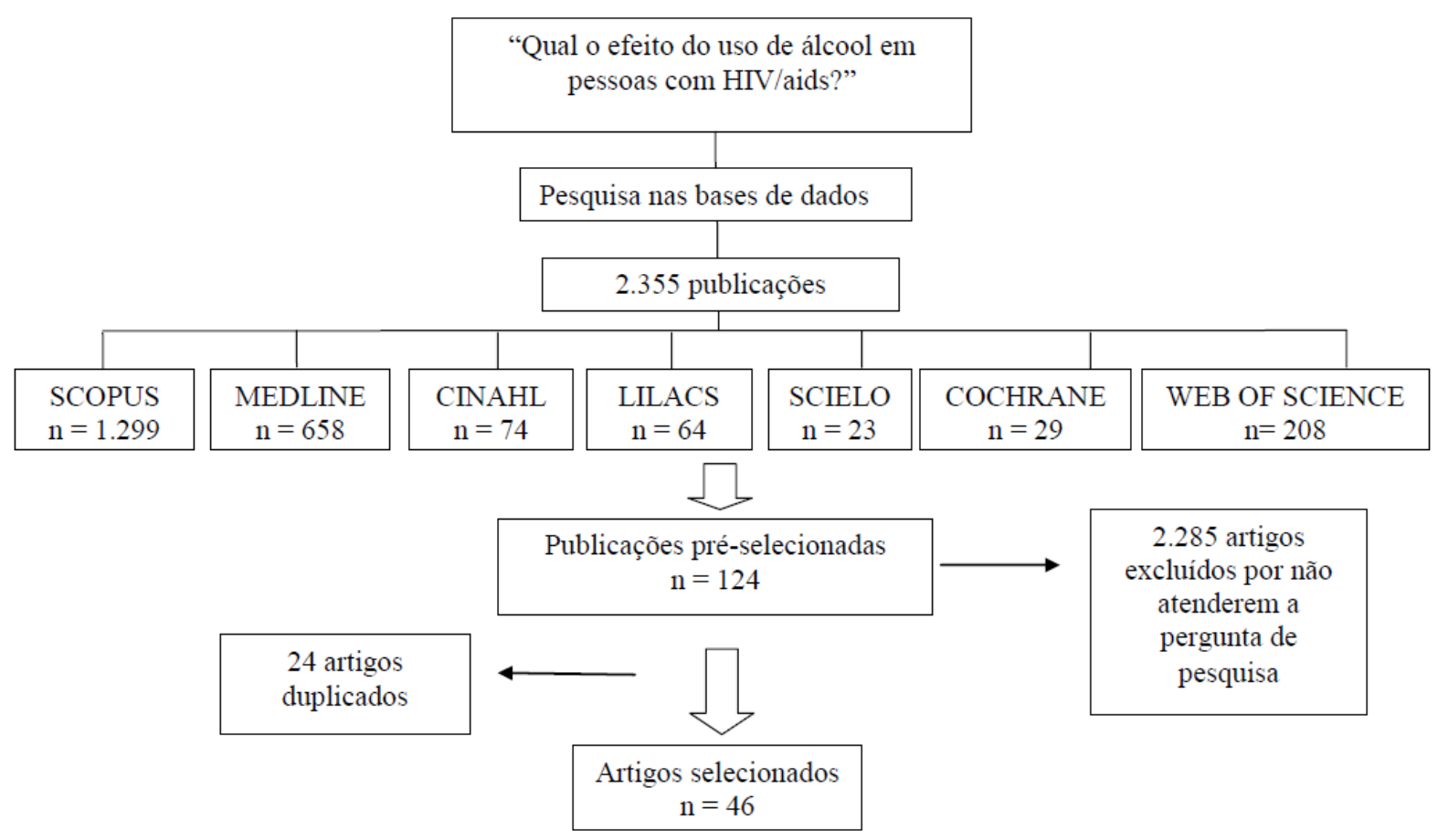

A análise dos dados foi realizada mediante tradução e leitura dos artigos na íntegra, por dois autores, de forma independente. Em caso de dúvidas, houve uma reunião entre os revisores para consenso. As informações foram transcritas e organizadas a partir de um instrumento validado ${ }^{(7)}$, que investigou: o desenvolvimento metodológico, os efeitos do uso de álcool em PVHA, a conclusão e os níveis de evidência.

Os níveis de evidência foram determinados da seguinte forma: Nível I Evidências provenientes de revisão sistemática ou meta-análise de múltiplos estudos clínicos controlados e randomizados ou oriundos de diretrizes clínicas, baseadas em revisões sistemáticas de ensaios clínicos controlados e randomizados; Nível II Evidências provenientes de estudos individuais controlados e randomizados; Nível III Evidências de estudos experimentais sem randomização; Nível IV Evidências provenientes de coorte ou caso-controle; Nível V Evidências oriundas de revisão sistemática de estudos descritivos e qualitativos; Nível VI Evidências originárias de um estudo descritivo ou qualitativo; Nível VII Evidências obtidas de opiniões de autoridades ou relatório de comitês de especialistas ${ }^{(8)}$.

Após análise, os efeitos do uso de álcool em PVHA foram agrupados em quatro categorias: 1.Efeitos neurológicos e metabólicos do álcool; 2.Efeitos do álcool na adesão à TARV; 3.Aumento do risco de transmissão do HIV e 4.Aumento da progressão da doença. Os achados foram discutidos com embasamento da literatura científica acerca da temática, sendo respeitada a integridade dos artigos e os direitos autorais, não havendo modificação do conteúdo encontrado em benefício desta pesquisa.

\section{RESULTADOS}

Quanto à caracterização dos artigos selecionados, o ano de publicação variou de 1993 a 2017, sendo 11publicados em países europeus ${ }^{(10,12,15-16,20,24,36-37,45,49,51)}, 28$ nos Estados Unidos ${ }^{(17-19,21-22,25-33,38-44,46-48,50,52-54)}$, 
quatro $^{(9,23,34-35)}$ no Brasil, dois em continente asiático ${ }^{(13-14)}$ e um ${ }^{(11)}$ no continente africano (Quadro 1). A análise dos níveis de evidência mostrou a seguinte distribuição: dois nível I ${ }^{(40-41)}$, dois nível II(17,26), 15 nível IV(15-16,27,30,3233,39,38,42-47,49,51-42), seis nível $\mathrm{V}^{(18,29,35-38)}$ e 21 nível VI(9-14,19-25,28,31,34,43,48,50,53-54).

Quadro 1: Caracterização dos estudos segundo o periódico, ano, país, tipo de estudo, desfecho em PVHA. Fortaleza/CE, 2017.

\begin{tabular}{|c|c|c|c|c|}
\hline Periódico & Ano & País & Tipo de estudo & Desfecho em PVHA \\
\hline BMC Infect Dis. ${ }^{(9)}$ & 2017 & Brasil & Estudo transversal & Baixa contagem de linfócitos T CD4+ \\
\hline Addict Behav.(10) & 2016 & Inglaterra & Estudo transversal & Aumento da sensibilidade à ansiedade \\
\hline PloS one..$^{(11)}$ & 2015 & África do Sul & Estudo transversal & Falha e interrupção do tratamento \\
\hline AIDS Care. ${ }^{(12)}$ & 2015 & Inglaterra & Estudo transversal & $\begin{array}{c}\text { Contagem de linfócitos T CD4+ inferiores a } 200 \\
\text { células } / \mathrm{mm}^{3}\end{array}$ \\
\hline AIDS Care. ${ }^{(13)}$ & 2015 & Rússia & Estudo transversal & Redução da adesão à TARV \\
\hline BMC Public Health(14) & 2014 & Vietnã & Estudo transversal & $\begin{array}{l}\text { Redução da adesão à TARV e da qualidade de } \\
\text { vida }\end{array}$ \\
\hline Curr Res HIV.(15) & 2014 & Holanda & Estudo de coorte & $\begin{array}{l}\text { Transtornos neurocognitivos como redução do } \\
\text { fator neurotrófico derivado do cérebro (BDNF) }\end{array}$ \\
\hline Brain Pathol.(16) & 2014 & Suíça & Estudo de coorte & $\begin{array}{c}\text { Níveis elevados dos marcadores de } \\
\text { neuroinflamação }\end{array}$ \\
\hline Subst Abus.(17) & 2014 & EstadosUnidos & Ensaio Clínico & Prevalência de sintomas depressivos \\
\hline Behav Med.(18) & 2014 & $\begin{array}{l}\text { Estados } \\
\text { Unidos }\end{array}$ & $\begin{array}{l}\text { Revisão de } \\
\text { Literatura }\end{array}$ & $\begin{array}{c}\text { Menor adesão à TARV e comportamento sexual } \\
\text { de risco }\end{array}$ \\
\hline $\begin{array}{l}\text { AIDS Res Hum } \\
\text { Retroviruses. }{ }^{(19)}\end{array}$ & 2014 & $\begin{array}{l}\text { Estados } \\
\text { Unidos }\end{array}$ & Estudo transversal & Baixa supressão virológica \\
\hline Int J Behav Med.(20) & 2014 & Geórgia & Estudo transversal & $\begin{array}{l}\text { Baixa supressão viral e menor adesão ao } \\
\text { tratamento }\end{array}$ \\
\hline Curr HIV Res.(21) & 2014 & $\begin{array}{l}\text { Estados } \\
\text { Unidos }\end{array}$ & Estudo transversal & $\begin{array}{l}\text { Progressão para estágio avançado/aids, baixa } \\
\text { contagem de linfócitos T CD4+, maior } \\
\text { susceptibilidade a infecções oportunistas e } \\
\text { menor adesão aos medicamentos }\end{array}$ \\
\hline Scientific World Journal.(22) & 2013 & $\begin{array}{l}\text { Estados } \\
\text { Unidos }\end{array}$ & Estudo transversal & Aumento da pressão arterial \\
\hline Brasília Med.(23) & 2013 & Brasil & Relato de caso & $\begin{array}{l}\text { Disfunção do sistema imunológico e } \\
\text { plaquetopenia, além do aumento da } \\
\text { suscetibilidade às doenças infecciosas e } \\
\text { oportunistas. }\end{array}$ \\
\hline HIV Med. ${ }^{(24)}$ & 2013 & Inglaterra & Estudo transversal & $\begin{array}{c}\text { Práticas sexuais de risco, como relação sexual } \\
\text { sem preservativo e com parceiros } \\
\text { sorodiscordantes }\end{array}$ \\
\hline $\begin{array}{l}\text { AIDS Res Hum } \\
\text { Retroviruses. }\end{array}$ & 2013 & $\begin{array}{l}\text { Estados } \\
\text { Unidos }\end{array}$ & Estudo transversal & $\begin{array}{l}\text { Maior risco de não adesão ao tratamento } \\
\text { farmacológico }\end{array}$ \\
\hline $\begin{array}{l}\text { J Acquir Immune Defic } \\
\text { Syndr.(26) }\end{array}$ & 2012 & $\begin{array}{l}\text { Estados } \\
\text { Unidos }\end{array}$ & Ensaio clínico & Efeitos tóxicos no organismo \\
\hline Biol Psychiatry. ${ }^{(27)}$ & 2012 & $\begin{array}{l}\text { Estados } \\
\text { Unidos }\end{array}$ & Estudo de coorte & $\begin{array}{l}\text { Déficit de volume cerebral mais profundo e } \\
\text { consistente }\end{array}$ \\
\hline J Subst Use. (28) & 2012 & $\begin{array}{l}\text { Estados } \\
\text { Unidos }\end{array}$ & Estudo Transversal & $\begin{array}{c}\text { Múltiplos parceiros, relações sexuais } \\
\text { desprotegidas }\end{array}$ \\
\hline $\begin{array}{l}\text { Expert Opin Drug Metab } \\
\text { Toxicol. (29) }\end{array}$ & 2012 & $\begin{array}{l}\text { Estados } \\
\text { Unidos }\end{array}$ & Revisão sistemática & $\begin{array}{l}\text { Aumento da toxicidade neuronal; redução da } \\
\text { adesão à TARV e da qualidade de vida; maior } \\
\text { supressão imune e uma progressão mais rápida } \\
\text { para a AIDS; exacerbação da neuroinflamação e } \\
\text { comprometimento neuropsicológico }\end{array}$ \\
\hline AIDS Care. ${ }^{(30)}$ & 2011 & $\begin{array}{l}\text { Estados } \\
\text { Unidos }\end{array}$ & Estudo de Coorte & Redução da adesão à TARV \\
\hline Subst Abus. & 2011 & $\begin{array}{l}\text { Estados } \\
\text { Unidos }\end{array}$ & Estudo Transversal & Não adesão à TARV \\
\hline
\end{tabular}




\begin{tabular}{|c|c|c|c|c|}
\hline Periódico & Ano & País & Tipo de estudo & Desfecho em PVHA \\
\hline Drug Alcohol Depend.(32) & 2011 & $\begin{array}{l}\text { Estados } \\
\text { Unidos }\end{array}$ & Estudo de Coorte & Sintomas depressivos \\
\hline Alcohol Clin Exp Res. ${ }^{(33)}$ & 2011 & $\begin{array}{l}\text { Estados } \\
\text { Unidos }\end{array}$ & Estudo de Coorte & Aumento da taxa de mortalidade \\
\hline J Bras Psiquiatr. & 2011 & Brasil & Estudo transversal & $\begin{array}{l}\text { Maior risco de não adesão ao tratamento } \\
\text { farmacológico }\end{array}$ \\
\hline J Bras Psiquiatr.(35) & 2010 & Brasil & Revisão sistemática & Redução da adesão à TARV \\
\hline Drug Alcohol Depend.(36) & 2010 & Irlanda & Revisão sistemática & Redução da adesão à TARV \\
\hline Alcohol Alcohol. ${ }^{(37)}$ & 2010 & Inglaterra & Revisão sistemática & Redução da adesão à TARV \\
\hline Curr HIV/AIDS Rep. (38) & 2010 & $\begin{array}{l}\text { Estados } \\
\text { Unidos }\end{array}$ & Revisão sistemática & Redução da adesão à TARV \\
\hline $\begin{array}{l}\text { Alcohol Research and } \\
\text { health. }{ }^{(39)}\end{array}$ & 2010 & $\begin{array}{l}\text { Estados } \\
\text { Unidos }\end{array}$ & Revisão sistemática & $\begin{array}{c}\text { Alargamento ventricular, redução de marcadores } \\
\text { de neurônios, além de dano importante na } \\
\text { bainha de mielina e degradação de feixes }\end{array}$ \\
\hline AIDS Behav. ${ }^{(40)}$ & 2009 & $\begin{array}{l}\text { Estados } \\
\text { Unidos }\end{array}$ & Revisão sistemática & Relações sexuais desprotegidas \\
\hline $\begin{array}{l}\text { J Acquir Immune Defic } \\
\text { Syndr. }{ }^{(41)}\end{array}$ & 2009 & $\begin{array}{l}\text { Estados } \\
\text { Unidos }\end{array}$ & Revisão sistemática & Baixa adesão à TARV \\
\hline AIDS Care. ${ }^{(42)}$ & 2009 & $\begin{array}{l}\text { Estados } \\
\text { Unidos }\end{array}$ & Estudo de Coorte & Sintomas depressivos \\
\hline AIDS Behav. ${ }^{(43)}$ & 2008 & $\begin{array}{l}\text { Estados } \\
\text { Unidos }\end{array}$ & Estudo transversal & $\begin{array}{c}\text { Relações sexuais desprotegidas e com múltiplos } \\
\text { parceiros }\end{array}$ \\
\hline $\begin{array}{l}\text { Addiction (Abingdon, } \\
\text { England). }{ }^{(44)}\end{array}$ & 2008 & $\begin{array}{l}\text { Estados } \\
\text { Unidos }\end{array}$ & Estudo de coorte & Sintomas depressivos \\
\hline Brain. (45) & 2007 & Inglaterra & Estudo de coorte & $\begin{array}{l}\text { Diminuição da anisotropia fracionada (FA) e } \\
\text { aumento na média de difusividade (MD) em } \\
\text { região calosa e feixes de fibras do cérebro }\end{array}$ \\
\hline J Stud Alcohol Drugs. $\stackrel{(46)}{ }$ & 2007 & $\begin{array}{l}\text { Estados } \\
\text { Unidos }\end{array}$ & Estudo de coorte & $\begin{array}{c}\text { Diminuição da qualidade de vida e aumento de } \\
\text { comorbidades psiquiátricas }\end{array}$ \\
\hline Neuroimage. ${ }^{(47)}$ & 2006 & $\begin{array}{l}\text { Estados } \\
\text { Unidos }\end{array}$ & Estudo de coorte & $\begin{array}{l}\text { Alargamento ventricular e aumento das regiões } \\
\text { frontais do cérebro }\end{array}$ \\
\hline $\begin{array}{l}\text { Journal of clinical and } \\
\text { experimental } \\
\text { neuropsychology. }{ }^{(48)}\end{array}$ & 2006 & $\begin{array}{l}\text { Estados } \\
\text { Unidos }\end{array}$ & Estudo transversal & Decréscimos no desempenho neurocognitivo \\
\hline $\begin{array}{l}\text { J Acquir Immune Defic } \\
\text { Syndr. }{ }^{(49)}\end{array}$ & 2003 & Inglaterra & Estudo de coorte & $\begin{array}{c}\text { Deficiência nutricional, metabólica e degradação } \\
\text { de células cerebrais }\end{array}$ \\
\hline J R Soc Med. ${ }^{(50)}$ & 1996 & $\begin{array}{l}\text { Estados } \\
\text { Unidos }\end{array}$ & Estudo Transversal & Progressão para estágio avançado/aids \\
\hline Alcohol Clin Exp Res. ${ }^{(51)}$ & 1995 & Inglaterra & Estudo de coorte & $\begin{array}{l}\text { Alterações metabólicas e cerebrais como a } \\
\text { redução de concentrações da substância branca } \\
\text { de fosfodiéster (PDE) e fosfocreatina (PCr) }\end{array}$ \\
\hline Biol Psychiatry. ${ }^{(52)}$ & 1995 & $\begin{array}{l}\text { Estados } \\
\text { Unidos }\end{array}$ & $\begin{array}{l}\text { Estudo de caso- } \\
\text { controle }\end{array}$ & $\begin{array}{c}\text { Danos nas células cerebrais e a morbidade do } \\
\text { Sistema Nervoso Central }\end{array}$ \\
\hline Clin Infect Dis. ${ }^{(53)}$ & 1994 & $\begin{array}{l}\text { Estados } \\
\text { Unidos }\end{array}$ & Estudo de caso & $\begin{array}{c}\text { Aumento da replicação viral e progressão para o } \\
\text { estágio mais avançado da doença }\end{array}$ \\
\hline $\begin{array}{l}\text { Neuropsiquiatria Clin } \\
\text { Neurosci. }{ }^{(54)}\end{array}$ & 1993 & $\begin{array}{l}\text { Estados } \\
\text { Unidos }\end{array}$ & Estudo Transversal & Efeitos neuropsicológicos \\
\hline
\end{tabular}

Em relação às categorias temáticas, houve predominância de artigos que abordaram os efeitos metabólicos e neurológicos do álcool (Categoria 1). Dentre esses efeitos, destacou-se a degradação de células cerebrais, com a consequente elevação dos marcadores de neuroinflamação, além dos transtornos neurocognitivos, como a demência e a falta de memória. Em relação às alterações metabólicas, destacou-se o déficit nutricional, com redução dos micronutrientes, tais como as vitaminas $A, B, C, D$ e $E$, além dos minerais zinco, ferro e selênio, 
influenciando diretamente na disfunção do sistema imunológico e na suscetibilidade às doenças infecciosas. O álcool também influenciou negativamente na qualidade de vida desses pacientes, causando redução na percepção dos domínios de qualidade de vida. No que concerne ao desenvolvimento de doenças, destacaram-se os transtornos depressivos e a hipertensão arterial sistêmica, além do aumento da sensibilidade à ansiedade. Ademais, constatou-se o risco de interações entre o álcool e outras drogas, o que pode ocasionar mais toxicidade ao organismo (Quadros 1 e 2).

Dezesseis estudos abordaram os efeitos do álcool na adesão à TARV (Categoria 2), demonstrando que o consumo de álcool reduz a capacidade de adesão ao regime terapêutico em PVHA, o que influencia negativamente na saúde, devido à redução dos linfócitos T CD4+ e aumento da carga viral (Quadros 1 e 2).

Com relação à categoria 3, foi demonstrado que o uso de álcool aumenta as chances de transmissão do vírus e a possibilidade de reinfecção, com o consequente aumento da resistência viral. Destaca-se o não uso de preservativos, atrelado ao consumo alcoólico durante as relações sexuais. Esses tipos de comportamentos de risco podem vir acompanhados da não revelação da sorologia positiva para o HIV ao companheiro, além da multiplicidade de parceiros (Quadros 1 e 2).

Acerca do aumento da progressão da doença (Categoria 4), identificou-se a elevação da carga viral e a redução da contagem de linfócitos T CD4+, os quais representam os marcadores de progressão da doença. Com isso, agrava-se o estado de saúde do paciente, direcionando-o para o estágio final da doença, podendo ocasionar a morte (Quadros 1 e 2).

Quadro 2: Quantidade de artigos que fundamentaram cada categoria temática, conforme assuntos abordados. Fortaleza/CE, 2017

\begin{tabular}{|c|c|}
\hline Categoria & Número de artigos \\
\hline Efeitos neurológicos e metabólicos do álcool & 21 \\
\hline Efeitos do álcool na adesão à TARV & 16 \\
\hline Aumento do risco de transmissão do HIV & 5 \\
\hline Aumento da progressão da doença & 9 \\
\hline TOTAL & $\mathbf{5 1}^{*}$ \\
\hline
\end{tabular}

* Nota: Alguns artigos foram classificados em mais de uma categoria.

\section{DISCUSSÃO}

A partir dessa pesquisa, foram descritos os efeitos do álcool no metabolismo, no sistema nervoso central, na adesão à TARV e sobre o aumento do risco de transmissão e progressão da doença em PVHA. A análise dos níveis de evidência mostrou predominância do nível VI, ou seja, as evidências provenientes de estudos descritivo ou qualitativos ${ }^{(8)}$.

Em referência aos efeitos neurológicos do consumo do álcool em PVHA, demonstrou-se que o álcool produz morbidade no sistema nervoso central, sobretudo, em regiões frontais do cérebro, que controlam as funções executivas e motoras ${ }^{(47)}$. Quando existe a comorbidade, esses efeitos tendem a se manifestar mais cedo, acarretando efeitos deletérios no córtex cerebral(52), como a degradação de células, a redução da massa branca ${ }^{(45)}$ e cinzenta do cérebro(51), redução da microestrutura calosal(45) e déficit no volume cerebral, principalmente nas regiões lateral, frontal, temporal, pariental e occipital, além do tálamo e corpo caloso ${ }^{(27)}$. Ademais, foi identificado alargamento ventricular, dano importante na bainha de mielina, redução nos marcadores de neurônios vivos ${ }^{(39,47)}$ e elevação dos marcadores de neuroinflamação(16). 
Evidências demonstram que os distúrbios neurológicos e cognitivos se acentuam ao longo dos anos, em decorrência do tempo de acometimento da doença, e quando há correlação com o álcool, esses efeitos se agravam ainda mais, trazendo danos consideráveis à saúde desses pacientes ${ }^{(15,36)}$. O uso exagerado de álcool em PVHA, com média de cinco a sete doses de bebida diária em quatro dias da semana, teve associação direta com o fator neurotrófico derivado do cérebro, quando comparado aos que não consomem bebidas alcoólicas ou aos que as utilizam esporadicamente, sendo um contribuinte significativo para o desenvolvimento de transtornos neurocognitivos associados ao HIV. Dentre esses transtornos associados, destacaram-se a demência e a falta de memória(15).

No que concerne ao desenvolvimento de doenças, destacou-se o risco de surgimento de sintomas depressivos, os quais podem estar associados à redução da adesão à TARV, e à progressão da doença(38,44). Ademais, há o risco adicional de desenvolvimento de hipertensão arterial sistêmica ${ }^{(33)}$ e de sensibilidade à ansiedade, a qual é considerada um fator de risco para o desenvolvimento de transtornos de ansiedade e depressão(10).

Com relação aos efeitos metabólicos, vale ressaltar ainda que o etilismo desempenha papel adverso significativo no volume do timo e na redução da contagem plaquetária. A plaquetopenia tem relação direta com a carga viral e a progressão da doença, estando associada com maior morbidade e mortalidade em virtude da deterioração dos linfócitos T CD4+ ${ }^{(23)}$, além do maior risco de desenvolvimento de anemia ${ }^{(49)}$.

Com relação ao efeito do álcool sobre a adesão à TARV, identificou-se que quanto maior o consumo de álcool, maior o índice de não adesão à TARV, o que leva à progressão e multiplicação do $\mathrm{HIV}^{(34,36-37,41)}$. Foi identificada diferença entre os gêneros e observou-se que as mulheres que ingerem álcool estão mais sujeitas a não aderirem ao esquema terapêutico, quando comparadas aos homens ${ }^{(35)}$.

Além disso, o consumo excessivo de álcool pode desenvolver interações com as medicações e alterar a proteína de ligação das drogas, onde o etanol compete com os fármacos nas ligações das isoenzimas do processo de metabolização. Com isso, esses consumidores podem ter maior risco de eventos adversos e de toxicidade pelos antirretrovirais e terapia ineficaz, quando comparado aos grupos em uso de placebo ${ }^{(26)}$, devido à concentração inadequada da droga no plasma ${ }^{(38)}$. Esses pacientes são mais vulneráveis socialmente, sendo necessárias estratégias de redução de danos para aumentar as chances de sucesso do uso da TARV(25).

Acerca dos efeitos do uso do álcool sobre o risco de transmissão do HIV, demonstrou-se que há um aumento do risco de reinfecção, disseminação e resistência viral, acarretando efeitos negativos na saúde desses indivíduos ${ }^{(37)}$. O álcool é considerado uma droga de fácil acesso e encontra-se presente em ocasiões de socialização e comemorações, onde há encontros entre parceiros sexuais, associando-se à redução do uso do preservativo em relações estáveis e casuais(55).

O consumo de álcool em PVHA pode acarretar a aquisição de comportamentos de risco, tais como as práticas sexuais desprotegidas e multiplicidade de parceiros ${ }^{(24,37,40)}$. Evidências demonstram que cada dose de álcool consumida aumenta em torno de $73 \%$ as chances de relações sexuais desprotegidas ${ }^{(52)}$, contudo, não se pode levar em consideração apenas o álcool como fator influenciador dessa prática, pois circunstâncias individuais e situacionais também estão envolvidas ${ }^{(43)}$. 
A respeito do impacto do uso dessa substância na progressão do HIV/aids, observou-se que o uso de álcool tem efeitos deletérios sobre a resposta imune, que podem influenciar no aumento de infecções, perturbações nas funções dos linfócitos B e ativação crônica de linfócitos T, que podem acelerar a progressão da doença, ocasionando maior suscetibilidade a infecções oportunistas como tuberculose, pneumonia bacteriana e hepatites virais $^{(16)}$. Além disso, produz efeitos sobre a transferência de bactérias intestinais que provocam ativação imunitária contra o HIV, resultando na progressão da doença( ${ }^{(38)}$.

Desse modo, consumo de álcool em PVHA tem forte associação com o aumento da morbidade e mortalidade desses indivíduos, visto que essa comorbidade tem relação direta com os marcadores de progressão da doença, fatores que influenciam negativamente nos domínios da qualidade de vida ${ }^{(46)}$. Evidências indicam que os consumidores de álcool tiveram nove vezes mais chances de apresentar contagens de linfócitos T CD4+ menores que 200 células $/ \mathrm{mm}^{3}$, sendo essa associação independente da adesão à TARV ${ }^{(12)}$. Além da influência na redução das células do sistema imune, destaca-se a relação com o aumento da carga viral(36-37), uma vez que o consumo de álcool pode suprimir o sistema imunológico e estimular a replicação do vírus ${ }^{(32,52,56)}$.

\section{CONCLUSÃO}

Essa revisão permitiu a identificação de vários efeitos negativos do consumo de álcool em PVHA, com destaque para as alterações neurológicas e metabólicas, adesão à TARV, aumento da transmissão do HIV e progressão da doença, indicando a necessidade de estratégias educativas para prevenção e redução do seu uso, que busquem identificar os principais fatores que levam ao consumo dessa substância, a fim de propor medidas direcionadas e cuidados individualizados.

Pesquisas com esse enfoque poderão auxiliar os profissionais de saúde e os enfermeiros a reconhecerem os impactos do uso do álcool nessa população, de maneira a instrumentalizá-los para a implementação de intervenções de acordo com o contexto de vida de cada paciente, buscando-se, quando necessário, acionar as redes sociais de apoio. Recomenda-se a realização de estudos que avaliem a eficácia de intervenções com esse propósito.

Uma limitação dessa revisão foi a ausência de pesquisas sobre os antirretrovirais que possuem interação com álcool, além de estudos que indicassem a diferença entre os efeitos do álcool na população feminina e masculina.

\section{REFERÊNCIAS}

1. Luo X, Duan S, Duan Q, Pu Y, Yang Y, Ding Y, et al. Alcohol use and subsequent sex among HIV-infected patients in an ethnic minority area of Yunnan Province, China. PLoS One. [Internet]. 2013 [cited 08 set 2016];8(4):e61660. Available from:

http://dx.doi.org/10.1371/journal.pone.0061660.

2. Molina PE, Bagby GJ, Nelson S. Biomedical consequences of alcohol use disorders in the HIV-infected host invited review. Curr Res HIV. [Internet]. 2014 [cited 08 set 2016];12(4):265-75. Available from:

https://www.ncbi.nlm.nih.gov/pmc/articles/PMC4222574/.

3. Orwat J, Saitz R, Tompkins CP, Cheng DM, Dentato MP, Samet JH. Substance abuse treatment utilization a mong adults living with HIV/AIDS and alcohol or drug problems. J Subst Abuso Treat. [Internet]. 2011 [cited 08 set 2016];41(3):233-42. Available from: http://dx.doi.org/10.1016/j.jsat.2011.04.002. 
4. Wandera B, Tumwesigye NM, Nankabirwa JI, Kambugu AD, Parkes-Ratanshi R, Mafigiri DK, et al. Alcohol consumption among HIV-infected persons in a large urban HIV clinic in Kampala Uganda: a constellation of harmful behaviors. PLoS One. [Internet]. 2015 [cited 08 set 2016];10(5):e0126236. Available from: http://dx.doi.org/10.1371/journal.pone.0126236.

5. Cooper HM. The integrative research review:a systematic approach. Beverly Hills (CA): Sage Publications; 1984. 6. Souza MT, Silva MD, Carvalho R. Integrative review: what is it? How to do it? Einstein. [Internet]. 2010 [cited 08 set 2016].8(1):102-6. Available from: http://dx.doi.org/10.1590/s1679-45082010rw1134.

7. Ursi ES. Prevenção de lesões de pele no perioperatório: revisão integrativa da literatura [dissertação]. [Ribeirão Preto]: Escola de Enfermagem, Universidade Federal de São Paulo; 2005. Available from: http://dx.doi.org/10.11606/D.22.2005.tde-18072005$\underline{095456 .}$.

8. Melnyk BM, Fineout-Overholt E. Making the case for evidence-based practice. In: Melnyk BM, Fineout-Overholt E, editors. Evidence-based practice in nursing \& healthcare: a guide to best practice. Philadelphia: Lippincott Williams \& Wilkins; 2011. 9. Silva CM, Mendoza-Sassi RA, Mota LD, Nader MM, Martinez AMB. Alcohol use disorders among people living with HIV/AIDS in Southern Brazil: prevalence, risk factors and biological markers outcomes. BMC infectious diseases. [Internet]. 2017 [cited 04 nov 2017];17(1):263. Available from: http://dx.doi.org/10.1186/s12879-017-2374-0.

10. Paulus DJ, Jardin C, Bakhshaie J, Sharp C, Woods SP, Lemaire C, et al. Anxiety sensitivity and hazardous drinking among persons living with HIV/AIDS: An examination of the role of emotion dysregulation. Addic Behav. [Internet]. 2016 [cited 08 set 2016];63:141-8. Available from: http://dx.doi.org/10.1016/j.addbeh.2016.07.013.

11. Kader R, Govender R, Seedat S, Koch JR, Parry C. Understanding the impact of hazardous and harmful use of alcohol and/or other drugs on ARV adherence and disease progression. PLoS One. [Internet]. 2015 [cited 04 nov 2017];10(5):e0125088. Available from: http://dx.doi.org/10.1371/journal.pone.0125088.

12. Malbergier A, Amaral RA, Cardoso LD. Alcohol dependence and CD4 cell count: Is there a relationship? AIDS Care. [Internet]. 2015 [cited 09 set 2016];27(1):54-8. Available from: http://dx.doi.org/10.1080/09540121.2014.947235.

13. Pecoraro A, Mimiaga M, O'Cleirigh C, Safren SA, Blokhina E, Verbitskaya E, Yaroslavtseva T, Ustinov A, Lioznov DA, Zvartau E, Krupitsky E, Woody GE. Depression, substance use, viral load, and CD4+ count among patients who continued or left antiretroviral therapy for HIV in St. Petersburg, Russian Federation. AIDS Care [Internet]. 2015 [cited 06 nov 2017];27(1):86-92. Available from: https://www.ncbi.nlm.nih.gov/pmc/articles/PMC4221462/.

14. Tran BX, Nguyen LT, Do CD, Nguyen QL, Maher RM. Associations between alcohol use disorders and adherence to antiretroviral treatment and quality of life amongst people living with HIV/AIDS. BMC Public Health. [Internet]. 2014 [cited 06 nov 2017];14(27):2-7. Available from: https://bmcpublichealth.biomedcentral.com/articles/10.1186/1471-2458-14-27.

15. Míguez-Burbano MJ, Espinoza L, Whitehead NE, Bryant VE, Vargas M, Cook RL, et al. Brain derived neurotrophic factor and cognitive status: the delicate balance among people living with HIV, with and without alcohol abuse. Curr Res HIV. [Internet]. 2014 [cited 08 set 2016];12(4):254-64. Available from: https://www.ncbi.nlm.nih.gov/pubmed/25053366.

16. Zarh NM, Mayer D, Rohlfing T, Sullivan E, Pfefferbaum A. Imaging Neuroinflammation? A perspective from MR Spectroscopy. Brain Pathol. [Internet]. 2014 [cited 09 set 2016];24(6):654-64. Available from: http://dx.doi.org/10.1111/bpa.12197.

17. Williams EC, Bradley KA, Balderson BH, McClure JB, Grothaus L, McCoy K, et al. Alcohol and associated characteristics among older persons living with human immunodeficiency virus on antiretroviral therapy. Substance abuse. [Internet]. 2014 [cited 04 nov 2017];35(3):245-253. Available from: http://dx.doi.org/10.1080/08897077.2014.890997.

18. Durvasula R, Miller TR. Substance abuse treatment in persons with HIV/AIDS: challenges in managing triple diagnosis. Behavioral Medicine. [Internet]. 2014 [cited 04 nov 2017];40(2):43-52. Available from:

http://dx.doi.org/10.1080/08964289.2013.866540.

19. Howe CJ, Cole SR, Napravnik S, Kaufman JS, Adimora AA, Elston B, et al.The role of at-risk alcohol/drug use and treatment in appointment attendance and virologic suppression among HIV(+) African Americans. [Internet]. 2014 [cited 04 nov 2017];30(3):233-240. Available from: http://dx.doi.org/10.1089/AID.2013.0163.

20. Kalichman SC, Grebler T, Amaral CM, McNerney M, White D, Kalichman MO, et al. Viral suppression and antiretroviral medication adherence among alcohol using HIV-positive adults. [Internet]. 2014 [cited 04 nov 2017];21(5):811-820. Availablefrom: http://dx.doi.org/10.1007/s12529-013-9353-7.

21. Agudelo M, Khatavkar P, Yndart A, Yoo C, Rosenberg R, G Devieux J, et al. Alcohol Abuse and HIV Infection: Role of DRD2. Current HIV research. [Internet]. 2014 [cited 04 nov 2017];12(4):234-242. Availablefrom:

https://www.ncbi.nlm.nih.gov/pubmed/25053368.

22. Ikeda MLR, Barcellos NT, Alencastro PR, Wolff FH, Brandão ABM, Fuchs FD, et al. Association of blood pressure and hypertension with alcohol consumption in HIV-infected white and nonwhite patients. Scientific World Journal. [Internet]. 2013 [cited 09 set 2016];(169825). Available from: http://dx.doi.org/10.1155/2013/169825.

23. Santos VM, Cruz LR, Paixão GTG, Mizuno CA, Teza ITV, Souza DWS. Plaquetopenia grave em paciente etilista com infecção por vírus da imunodeficiência humana. Brasília Med. [Internet]. 2013 [cited 10 set 2016];49(3):id672195. Available from:

http://bases.bireme.br/cgi-

bin/wxislind.exe/iah/online/?IsisScript=iah/iah.xis\&src=google\&base=LILACS\&lang=p\&nextAction=Ink\&exprSearch=672195\&indexS earch=ID. 
24. Kalichman SC, Grebler T, Amaral C, Mckerey M, White D, Kalichman M, et al. Assumed infectiousness, treatment adherence and sexual behaviours: Applying the swiss statement on infectiousness to HIV-positive alcohol drinkers. HIV Med. [Internet]. 2013 [cited 08 set 2016];14(5):263-72. Available from: http://dx.doi.org/10.1111/i.1468-1293.2012.01062.x.

25. Teixeira C, Dourado MDL, Santos MP, Brites C. Impact of use of alcohol and illicit drugs by AIDS patients on adherence to antiretroviral therapy in Bahia, Brazil. AIDS Res Hum Retroviruses. [Internet]. 2013 [cited 10 set 2016];29(5):799-804. Available from: http://dx.doi.org/10.1089/AID.2012.0296.

26. Mccance-Katz EF, Lum PJ, Beatty G, Gruber VA, Peters M, Rainey PM. Untreated HIV infection is associated with higher blood alcohol levels. J Acquir Immune Defic Syndr. [Internet]. 2012 [cited 10 set 2016];60(3):282-288. Available from:

http://dx.doi.org/10.1097/QAl.0b013e318256625f.

27. Pfefferbaum A, Rosenbloom, MJ, Sassoon SA, Kemper CA, Deresinski S, Rohlfing T, et al. Regional brain structural dysmorphology in human immunodeficiency virus infection: Effects of acquired immune deficiency syndrome, alcoholism, and age. Biol Psychiatry. [Internet]. 2012 [cited 08 set 2016].72(5):361-70. Available from:

http://dx.doi.org/10.1016/j.biopsych.2012.02.018.

28. Gerbi GB, Habtemariam T, Tameru B, Nganwa D, Robnett V. A comparative study of substance use before and after establishing HIV infection status among people living with HIV/AIDS Journal of substance use. [Internet]. 2011 [cited 04 nov 2017];16(6):464-

475. Available from: http://dx.doi.org/10.3109/14659891.2010.495820.

29. Kumar S, Jin M, Ande A, Sinha N, Silverstein PS, Kumar A. Alcohol consumption effect on antiretroviral therapy and HIV-1 pathogenesis: role of cytochrome P450 isozymes. Expert Opin Drug Metab Toxicol. [Internet]. 2012 [cited 06 nov 2017];8(11):136375. Available from: https://www.ncbi.nlm.nih.gov/pmc/articles/PMC4033313/.

30. Cohn SE, Jiang H, McCutchan JA, Koletar SL, Murphy RL, Robertson KR, de St Maurice AM, Currier JS, Williams PL. Association of ongoing drug and alcohol use with non-adherence to antiretroviral therapy and higher risk of AIDS and death: results from ACTG 362. AIDS Care [Internet]. 2011 [cited 06nov2017];23(6):775-85. Available from:

https://www.ncbi.nlm.nih.gov/pmc/articles/PMC3095689/.

31. Broyles LM, Gordon AJ, Sereika SM, Ryan CM, Erlen JA. Predictive Utility of Brief Alcohol Use Disorders Identification Test (AUDIT) for human immunodeficiency virus antiretroviral medication nonadherence. Substance abuse. [Internet]. 2011 [cited 04 nov 2017];32(4):252-261. Available from: http://dx.doi.org/10.1080/08897077.2011.599255.

32. Sullivan LE, Goulet JL, Justice AC, Fiellin DA. Alcohol consumption and depressive symptoms over time: a longitudinal study of patients with and without HIV infection. Drug and alcohol dependence. [Internet]. 2011 [cited 04 nov2017];117(2):158-163. Available from: http://dx.doi.org/10.1016/i.drugalcdep.2011.01.014.

33. DeLorenze GN, Weisner C, Tsai AL, Satre DD, QuesenberryJr CP. Excess mortality among HIV-infected patients diagnosed with substance use dependence or abuse receiving care in a fully integrated medical care program. Alcoholism: Clinicaland Experimental Research. [Internet]. 2011 [cited 04 nov 2017];35(2):203-210. Available from: http://dx.doi.org/10.1111/j.1530-0277.2010.01335.x. 34. Rego SRB, Oliveira CFA, Rego DMS, Júnior RFS, Silva VB. Estudo do autorrelato de adesão e uso problemático de álcool em uma população de indivíduos com aids em uso de HAART. J Bras Psiquiatr. [Internet]. 2011 [cited 10 set 2016];60(1):46-9. Available from: http://dx.doi.org/10.1590/S0047-20852011000100009.

35. Rego SEM, Rego DMS. Associação entre uso de álcool em indivíduos com aids e adesão ao tratamento antirretroviral: Uma revisão da literatura. J Bras Psiquiatr. [Internet]. 2010 [cited 08 set 2016];59(1):70-3. Available from:

http://dx.doi.org/10.1590/S0047-20852010000100011.

36. Azar MM, Springer SA, Meyer JP, Altice FL. A systematic review of the impact of alcohol use disorders on HIV treatment outcomes, adherence to antiretroviral therapy and health care utilization. Drug Alcohol Depend. [Internet]. 2010 [cited 08 set 2016]:112(3):178-93. Available from: http://dx.doi.org/10.1016/j.drugalcdep.2010.06.014.

37. Shuper PA, Neuman M, Kanteres F, Baliunas D, Joharchi N, Rehm J. Causal considerations on alcohol and HIV/AIDS - A systematic review. Alcohol Alcohol. [Internet]. 2010 [cited set 2016];45(2):159-166. Available from:

http://dx.doi.org/10.1093/alcalc/agp091.

38. Hahn JA, Samet JH. Alcohol and HIV disease progression: weighing the evidence. Curr HIV/AIDS Rep. [Internet]. 2010 [cited 08 set 2016];7(4):226-33. Available from: http://dx.doi.org/10.1007/s11904-010-0060-6.

39. Rosenbloom MJ, Sullivan EV, Pfefferbaum A. Focus on the brain: HIV infection and alcoholism comorbidity effects on brain structure and function. Alcohol Research e health. [Internet]. 2010 [cited 08 set 2016];33(3):247-57. Available from:

https://www.ncbi.nlm.nih.gov/pmc/articles/PMC3860510/.

40. Shuper PA, Joharchi N, Irving H, Rehm J. Alcohol as a correlate of unprotected sexual behavior among people living with HIV/AIDS: Review and meta-analysis. AIDS Behav. [Internet]. 2009 [cited 09 set 2016];13(6):1021-36. Available from:

http://dx.doi.org/10.1007/s10461-009-9589-z.

41. Hendershot CS, Stoner SA, Pantalone DW, Simoni JM. Alcohol use and antiretroviral adherence: Review and meta-analysis. J Acquir Immune DeficSyndr. [Internet]. 2009 [cited 10 set 2016];52(2):180-202. Available from: http://dx.doi.org/10.1097/QAl.0b013e3181b18b6e. 
42. Ghebremichael M, Paintsil E, Ickovics JR, Vlahov D, Schuman P, Boland R, et al. Longitudinal association of alcohol use with HIV disease progression and psychological health of women with HIV. AIDS care. [Internet]. 2009 [cited 04 nov 2017];21(7):834-841. Available from: http://dx.doi.org/10.1080/09540120802537864.

43. Barta WD, Portnoy DB, Kiene SM, Tennen H, Abu-Hasaballah KS, Ferrer R. A daily process investigation of alcohol-involved sexual risk behavior among economically disadvantaged problem drinkers living with HIV/AIDS. AIDS Behav. [Internet]. 2008 [cited 09 set 2016];2(5):729-40. Available from: http://dx.doi.org/10.1007/s10461-007-9342-4.

44. Sullivan LE, Saitz R, Cheng DM, Libman H, Nunes D, Samet JH. The impact of alcohol use on depressive symptoms in HIVinfected patients. Addiction (Abingdon, England).[Internet]. 2008 [cited em 10 set 2016];103(9):1461-1467. Available from: http://dx.doi.org/10.1111/j.1360-0443.2008.02245.x.

45. Pfefferbaum A, Rosenbloom MJ, Adalsteinsson E, Sullivan EV. Diffusion tensor imaging with quantitative fibre tracking in HIV infection and alcoholism comorbidity: synergistic white matter damage. Brain. [Internet]. 2007 [cited 09 set 2016];130(Pt 1):48-64. Available from: http://dx.doi.org/10.1093/brain/awl242.

46. Rosenbloom MJ, Sullivan EV, Sassoon SA, O'Reilly A, Fama R, Kemper CA, et al. Alcoholism, HIV infection, and their comorbidity: factors affecting self-rated health-related quality of life. J Stud Alcohol Drugs. [Internet]. 2007 [cited 10 set 2016];68(1):115-25. Available from: https://www.ncbi.nlm.nih.gov/pubmed/17149525.

47. Pfefferbaum A, Rosenbloom MJ, Rohlfing T, Adalsteinsson E, Kemper CA, Deresinski S, et al. Contribution of alcoholism to brain dysmorphology in HIV infection: Effects on the ventricles and corpus callosum. Neuroimage. [Internet]. 2006 [cited 09 set 2016];33(1):239-51. Available from: http://dx.doi.org/10.1016/j.neuroimage.2006.05.052.

48. Durvasula RS, Myers HF, Mason K, Hinkin C. Relationship between alcohol use/abuse, HIV infection and neuropsychological performance in African American men. Journal of clinical and experimental neuropsychology. [Internet]. 2006 [cited 04 nov 2017];28(3):383-404. Available from: http://dx.doi.org/10.1080/13803390590935408.

49. Conigliaro J, Gordon AJ, Mcginnis KA, Rabeneck L, Justice AC. How harmful is hazardous alcohol use and abuse in HIV infection: Do health care providers know who is at risk? J Acquir Immune DeficSyndr. [Internet]. 2003 [cited 08 set 2016];33(4):521-25.

Available from: https://www.ncbi.nlm.nih.gov/pubmed/12869842.

50. Lake-Bakaar G, Grimson R. Alcohol abuse and stage of HIV disease in intravenous drug abusers. Journal of the Royal Society of Medicine. [Internet]. 1996 [cited 04 nov 2017];89(7):389-392. Available from:

https://www.ncbi.nlm.nih.gov/pmc/articles/PMC1295853/.

51. Meyerhoff DJ, Mackay S, Sappey-Marinier D, Deicken R, Calabrese G, Dillon WP, et al. Effects of chronic alcohol abuse and HIV infection on brain phosphorus metabolites. Alcohol Clin Exp Res. [Internet]. 1995 [cited 09 set 2016];19(3):685-92. Available from: https://www.ncbi.nlm.nih.gov/pubmed/7573794.

52. Fein G, Biggins CA, Mackay S. Alcohol abuse and HIV infection have additive effects on frontal cortex function as measured by auditory evoked potential P3A latency. Biol Psychiatry. [Internet]. 1995 [cited 10 set 2016];37(3):183-95. Available from: http://dx.doi.org/10.1016/0006-3223(94)00119-N.

53. Fong IW, Read S, Wainberg MA, Chia WK, Major C. Alcoholism and rapid progression to AIDS after seroconversion. Clin Infect Dis. [Internet].1994 [cited 10 set 2016];19(2):337-8. Available from: https://www.ncbi.nlm.nih.gov/pubmed/7986912.

54. Bornstein RA, Fama R, Rosenberger P, Whitacre CC, Para MF, Nasrallah HA, et al. Drug and alcohol use and neuropsychological performance in asymptomatic HIV infection. Journal of Neuropsychiatry and Clinical Neurosciences, [Internet]. 1993 [cited 04 nov 2017];5:254-254. Available from: http://dx.doi.org/10.1176/inp.5.3.254.

55. Barta WD, Tennen H, Kiene SM. Alcohol-involved sexual risk behavior among heavy drinkers living with HIV/AIDS: negative affect, self-efficacy, and sexual craving. Psychol Addict Behav. [Internet]. 2010 [cited 08 set 2016];24(4):563-70. Available from: http://dx.doi.org/10.1037/a0021414

56. Bagby GJ, Zhang P, Purcell JE, Didier PJ, Nelson S. Chronic binge ethanol consumption accelerates progression of simian immunodeficiency virus disease. Alcohol Clin Exp Res. [Internet]. 2006 [cited 09 set 2016];30(10):1781-90. Available from: http://dx.doi.org/10.1111/j.1530-0277.2006.00211.x. 